\title{
Mobilidade pendular e a integração metropolitana: uma proposta metodológica para os municípios da Região Metropolitana de Belo Horizonte/MG - 2010*
}

\author{
Carlos Lobo ${ }^{\star \star}$ \\ Leandro Cardoso ${ }^{\star \star \star}$ \\ Ivânia Linhares de Almeida \\ Ricardo Alexandrino Garcia ${ }^{\star \star \star \star \star}$
}

Não é raro na literatura acadêmica específica haver controvérsias sobre os limites e a própria configuração territorial das regiões metropolitanas no Brasil. Afora a falta de consenso e critérios bem definidos para o estabelecimento desses recortes regionais e político-administrativos, parece relevante considerar o significado da mobilidade pendular como indicador da dinâmica econômica e social metropolitana. 0 objetivo desse artigo é investigar o nível de integração dos municípios que compõem a Região Metropolitana de Belo Horizonte, tendo como base os fluxos de deslocamentos pendulares. Com informações extraídas dos microdados amostrais do Censo Demográfico 2010, a metodologia apresentada permitiu a proposição, para cada município da região, de um Índice de Integração Regional, derivado da razão de pendularidade interna, razão de conectividade pendular e razão de pendularidade nuclear. Em geral, os resultados indicaram sensíveis diferenças regionais no espaço metropolitano, o que permitiu destacar alguns níveis muito baixos de integração, especialmente para os casos dos municípios de Itatiaiuçu, Itaguara e Baldim.

Palavras-chave: Integração metropolitana. Mobilidade pendular. Região Metropolitana de Belo Horizonte.

\footnotetext{
* Os autores agradecem ao CNPq e à Fapemig pelo auxílio financeiro aos projetos de pesquisa. Cabe ainda menção ao apoio oferecido pela Fapemig por meio da concessão de bolsa de pesquisa pelo Programa Pesquisador Mineiro.

** Instituto de Geociências (IGC), Universidade Federal de Minas Gerais (UFMG), Belo Horizonte-MG, Brasil (carlosfflobo@ gmail.com).

${ }^{* \star \star}$ Escola de Engenharia, Universidade Federal de Minas Gerais (UFMG), Belo Horizonte-MG, Brasil (leandro@etg.ufmg.br). ${ }^{\star \star \star \star}$ Universidade Federal de Minas Gerais (UFMG), Belo Horizonte-MG, Brasil (ivanialinhares@gmail.com).

***** Instituto de Geociências (IGC), Universidade Federal de Minas Gerais (UFMG), Belo Horizonte-MG, Brasil (alexandrinogarcia@gmail.com).
} 


\section{Introdução}

A relativa fragilidade na definição dos critérios técnicos para a criação, no Brasil, das nove Regiões Metropolitanas (RMs) na década de 1970, institucionalizadas sob um regime autoritário e centralizador, não foi superada com a promulgação da Constituição Federal de 1988. Não obstante seu caráter democrático, que transformou os municípios em entes federados com maior autonomia e concedeu aos Estados o poder de criar suas próprias RMs, a Carta Magna brasileira não estabeleceu as diretrizes específicas para institucionalização dessas regiões. Na verdade, essa lacuna legal manteve o descompasso entre a configuração do território metropolitano e a dinâmica socioeconômica regional, o que, não raro, dificultou a gestão e o planejamento integrado, bem como a implantação de políticas públicas regionais eficazes, inclusive aquelas voltadas para a maior eficiência e qualidade na mobilidade espacial da população. Em 12 de janeiro de 2015, foi sancionada a Lei Federal n. 13.089, denominada Estatuto da Metrópole, que representou um importante marco regulatório para suprir o vácuo deixado pela Constituição de 1988, no que diz respeito tanto às diretrizes para a criação de RMs, quanto ao planejamento, gestão e governança interfederativa nas RMs e Aglomerações Urbanas (AUs). Ainda que os dispositivos do Estatuto da Metrópole pudessem contribuir para o entendimento da nova estrutura de gestão para o planejamento das funções públicas de interesse coletivo, há ainda obstáculos para a efetiva implementação das diretrizes gerais de institucionalização regional, especialmente aqueles de natureza política.

Com efeito, dentre as variáveis que tradicionalmente servem de parâmetro para a identificação do grau de integração intermunicipal metropolitana, destacam-se os chamados movimentos pendulares que, além da intensidade e direção dos fluxos populacionais diários no tecido metropolitano, refletem a dinâmica e organização do mercado laboral e educacional. A mobilidade intrametropolitana configura-se, portanto, como um indicador sensível ao grau de interação municipal no espaço regional, refletindo, dessa forma, a própria densidade social do espaço metropolitano. No caso da Região Metropolitana de Belo Horizonte (RMBH), assim como nos principais aglomerados metropolitanos no Brasil, os deslocamentos diários de população têm sido resultantes, principalmente, do movimento do capital imobiliário juntamente com ações seletivas do Estado na dotação de infraestrutura econômica e programas habitacionais. 0 objetivo deste trabalho é, portanto, avaliar, com base em indicadores derivados da dinâmica pendular metropolitana, o nível de integração dos municípios que integram a RMBH. Para esse propósito, foram utilizados os microdados amostrais do Censo Demográfico 2010, tendo como base as variáveis que identificam o município de residência e aquele de trabalho ou estudo.

\section{A dispersão urbana e a integração municipal metropolitana: conceito e significado dos movimentos pendulares}

Não são novas as incursões teóricas sobre o significado e as características do processo de urbanização no Brasil, bem como seus impactos nas condições de vida da população 
e nas formas e arranjos espaciais, mais ou menos dispersos. Como destacou Reis (2006), o conteúdo da urbanização varia em diferentes níveis espaciais e envolve diversidades e articularidades em espaços intraurbanos e regionais, o que requer a incorporação da análise das relações entre centro e periferia, em suas múltiplas escalas. 0 processo de dispersão urbana, como descreve o autor na mesma obra, caracteriza-se pelo esgarçamento do tecido urbano, com a urbanização estendendo-se pelo território, com a inclusão de núcleos urbanos separados por vazios intersticiais, mantendo vínculos estreitos e um único sistema urbano. Formam-se constelações ou nebulosas de núcleos urbanos de diferentes dimensões, integrados às aglomerações urbanas metropolitanas e submetropolitanas, com o sistema de vias de transporte inter-regionais utilizado como apoio ao transporte diário (REIS, 2006).

Para Limonad (2011), embora haja uma difusão da dispersão urbana em diversas partes do mundo, ainda persistem formas de urbanização intensiva. Ambas, apesar de levarem a uma expansão territorial da malha urbana, diferem na forma final que essa expansão assume. Ao tecido urbano esgarçado, fragmentado e pulverizado da urbanização extensiva, característico do que Francesco Indovina denominou de arquipélago metropolitano, contrapõem-se grandes aglomerações urbanas com alta densidade, onde se afirma a primazia da metrópole e da cidade concentrada e compacta. No Brasil essa dispersão assume diversas manifestações quanto à localização espacial, seja como forma de expansão das periferias metropolitanas, pela multiplicação dispersa de pequenos aglomerados urbanos em bacias de emprego, seja sob a forma de clusters industriais, de serviços ou turísticos acompanhados, muitas vezes, pela formação de condomínios ou megacondomínios fora das áreas urbanas (LIMONAD, 2011).

Afora as controvérsias teóricas e as evidências empíricas trazidas sobre a abrangência da dispersão urbana no caso brasileiro, parece não haver dúvidas sobre a necessidade de uma maior reflexão sobre as particularidades de cada uma das regionais metropolitanas do país, inclusive aquelas referentes à mobilidade espacial da população. Cruz (2010) acredita que a definição de Região Metropolitana como recorte para execução de políticas públicas apresenta obstáculos, tendo em vista que existem expressivas diferenças internas, inclusive no nível de interação entre os municípios metropolitanos. Para o autor, existe um impasse com relação à unidade (recorte) adequada para o planejamento no âmbito metropolitano. Na maioria das vezes, parte-se de um recorte territorial definido a priori e não de uma análise na qual os deslocamentos pendulares definam a extensão da área a ser considerada dispersa (OJIMA, 2011). Pode-se, dessa forma, como apresenta Ojima (2011), seguir duas opções: predefinir qual é o limite territorial que estamos tratando (município A, região metropolitana $\mathrm{B}$, etc.) e buscar analisar a dispersão urbana e os deslocamentos populacionais dentro desse recorte; ou definir, a partir dos deslocamentos populacionais, a extensão do território que deverá ser considerado o recorte de estudo.

Como demonstrado por Branco (2003), existe uma tendência de que nos espaços mais adensados os relacionamentos com o core metropolitano sejam mais intensos. Portanto, a 
densificação territorial potencializa as relações socioeconômicas no âmbito metropolitano, o que pode contribuir para a redução dos custos de transportes e da sua infraestrutura, potencializando a mobilidade espacial da população. ${ }^{1} \mathrm{~A}$ chamada mobilidade pendular passa a ser um dos fenômenos característicos da realidade metropolitana, que, conforme destacou Jardim (2011), reflete diretamente a expansão do território metropolitano, indicando, do ponto de vista social e geográfico, a emersão de novas centralidades. Como sugerem Moura et al. (2005), a intensidade da pendularidade populacional é um importante indicador da extensão da urbanização no território, constituindo uma informação útil à delimitação de grandes áreas urbanas. ${ }^{2} 0$ próprio Bureau do Censo dos Estados Unidos utiliza o movimento pendular por motivo de trabalho na definição e delimitação de grandes conjuntos metropolitanos. Na França, o Institut Natitonal de la Statistique et des Etudes Economiques usa esse mesmo indicador na identificação de polarização de uma área urbana (JULIEN, 2000).

Conceitualmente, a mobilidade pendular se refere, de forma genérica, ao movimento realizado por indivíduos que se deslocam entre unidades espaciais, delimitadas pelo seu local de residência e o de trabalho ou estudo. Essas unidades espaciais frequentemente são tomadas por municípios relativamente próximos ou limítrofes, o que envolve normalmente deslocamentos de curta distância. Contudo, nesses trajetos menos distantes podem ser necessárias horas ao deslocamento, o que pode ocorrer, por exemplo, no caso de deslocamentos entre municípios que não contam com vias/estradas de boa qualidade ou com meios de transporte pouco eficientes, que tenham trechos com alto grau de congestionamento dada a existência de áreas de alta densidade populacional.

Ainda que seja conceitualmente distinta dos movimentos migratórios, a mobilidade pendular apresenta reflexos e efeitos similares demograficamente, uma vez que a unidade espacial de referência experimenta um acréscimo populacional temporário, que pode ser significativo em determinados momentos do dia. De acordo com Aranha (2005, p. 96),

Do ponto de vista demográfico, os deslocamentos pendulares modificam provisoriamente o volume populacional do município e aumentam ou diminuem seu tamanho dependendo das características de cada área. Assim, se o município for mais concentrador ou mais dispersor, os deslocamentos podem gerar maior ou menor demanda por serviços ou bens ou aumentar sua oferta para a população residente.

\footnotetext{
${ }^{1}$ Como demonstrado por Lobo (2016, p. 296), de modo geral, a análise sobre a mobilidade pendular no Brasil, identificada pelo Censo Demográfico de 2010, indica dois pontos centrais de conclusões: “1ํ) há um importante incremento na mobilidade pendular envolvendo as principais metrópoles brasileiras e os municípios de suas respectivas Unidades da Federação; $\left.2^{9}\right)$ que esse crescimento foi predominante direcionado para as periferias metropolitanas, embora tenha ocorrido um crescimento no volume e no número de municípios mais distantes que abrigam populações que trabalham ou estudam no core metropolitano".

${ }^{2}$ Assim, como demonstraram Frey e Speare Jr. (1992), “essas transformações na forma de assentamento definiriam um novo conceito de “área metropolitana”, como "forma ampliada de vida local caracterizada por alta densidade de movimento pendular, que passa a ser o principal indicador do espaço de atividade da comunidade. A discussão do movimento é, portanto, indissociável da de mobilidade, que vai caracterizar a vida urbana atual, acentuando a importância dos transportes, sobretudo o individual” (MOURA et al., 2005, p. 122).
} 
A pendularidade também pode envolver outro tipo de configuração urbana: aquela que diz respeito à mobilidade de famílias/pessoas de alta renda, que residem nos chamados condomínios fechados e se deslocam diariamente para outros municípios para trabalhar ou estudar. Esse fenômeno é, dentre outros aspectos, decorrente da busca por uma esperada melhor qualidade de vida, especialmente por esperadas amenidades ambientais, incluindo a suposta condição de menor violência em determinados municípios de menor porte. Trata-se de um movimento essencialmente residencial, sem, contudo, haver alteração no local de trabalho. Como esclarecem Jardim e Ervatti (2007, p. 7),

A mobilidade residencial refere-se aos deslocamentos da população no interior de uma determinada unidade administrativa tendo como unidade mínima de referência o município, pelo fato que a mudança de residência não implica, necessariamente, mudança de emprego ou atividade.

Pode-se, sobre esse aspecto, considerar que a pendularidade seja uma consequência da mobilidade metropolitana determinada pela ampliação dos vetores de expansão do tecido urbano do core metropolitano. Isso reflete escolhas residenciais de segmentos da população na expectativa de que externalidades positivas compensam os custos adicionais da distância ao trabalho, ou, no caso dos trabalhadores de baixa renda, a ação excludente do mercado imobiliário e/ou do mercado de trabalho (BRITO; SOUZA, 2005). Se alguns municípios se "beneficiam" do aumento provisório da população, dado o incremento da demanda de vários serviços locais, outros apresentam uma condição bem diferenciada fora do turno laboral, característica das conhecidas "cidades-dormitório", o que foi objeto de preocupação em face de condição "pouco animada" do ponto de vista urbanístico, como infraestrutura urbana precária, parcelamentos irregulares e alto volume de trabalhadores pouco qualificados, que, marcada por uma "paisagem dominada por moradias inacabadas, produto da autoconstrução ou mutirão, é algo bastante conhecido nas periferias das metrópoles brasileiras" (LOBO, 2016, p. 290). ${ }^{3}$

Para Ojima (2007, p. 126), pode-se supor "que o padrão de cidades-dormitório com baixo dinamismo econômico só passa a existir quando a proporção de movimentos pendulares é mais elevada, em torno de $40 \%$ da população ocupada". Ao considerar o percentual da população economicamente ativa ocupada entre 15 e 64 anos que se desloca para o núcleo metropolitano, podem-se considerar alguns exemplos de municípios-dormitório da RMBH: Ibirité (51\%), Ribeirão das Neves (57\%), Sabará (63\%), Santa Luzia (50\%) e Vespasiano (49\%), conforme dados do Censo Demográfico de 2010 (OJIMA, 2010). Há, segundo Pereira e Herrero (2009, p. 22), "fluxos pendulares do tipo puro desconcentração produtiva”, indicando “um possível processo de integração metropolitana multipolarizada,

\footnotetext{
${ }^{3}$ Há, como esclarece Ojima (2010, p. 398), “uma associação quase que imediata do processo de metropolização ao surgimento das ‘cidades-dormitório' baseado no modelo dicotômico ‘centro-periferia’”.
} 
reforçando a dinamização de subcentros econômicos da região e apontando novos eixos de expansão urbana e demanda viária". ${ }^{4}$

Lobo et al. (2014), com base nos dados referentes à mobilidade pendular na RMBH, utilizando as bases dos Censos Demográficos de 2000 e 2010, avaliaram o nível de centralidade e de dispersão espacial metropolitana. Os resultados apresentados apontaram para uma pequena redução na proporção dos fluxos diários do tipo residência/trabalho no sentido periferia/núcleo e um crescimento mais expressivo, tanto absoluto como relativo, dos deslocamentos na direção núcleo/periferia (denominados de pendularidade inversa). Como exemplo, verifica-se que o município de Confins teve um acréscimo superior a $300 \%$. Destacam-se, ainda, tanto em termos relativos como absolutos, Nova Lima e Betim, que apresentaram um incremento em torno de $50 \%$. As evidencias sugerem que parece ocorrer o fortalecimento de determinadas centralidades, além do core metropolitano, que tem induzido uma mobilidade ampliada no espaço metropolitano (LOBO et al., 2014). Verifica-se, em geral, que o core metropolitano, mesmo que a mobilidade na periferia tenha experimentado um incremento considerável, mantém sua influência como fornecedor de postos de trabalho e serviços, principalmente para os ditos “municípios-dormitório”. Dessa forma, a pendularidade pode servir como um importante parâmetro de apoio à gestão da mobilidade metropolitana. Como indicador-síntese da interação socioeconômica entre municípios, a pendularidade retrata, em grande medida, a mobilidade da força de trabalho e da população em atividade escolar, não raro consideradas variáveis definidoras do nível de polarização e influência regional (CASTELLO BRANCO; FIRKOWSKI; MOURA, 2005; DURANTON, 2013; OJIMA, 2011).

\section{o Índice de Integração Regional para a RMBH: o recorte de análise e a metodologia proposta}

A RMBH foi criada pela Lei Complementar Federal n. 14, de 8 de junho de 1973. Era inicialmente composta pelos municípios de Belo Horizonte, Betim, Caeté, Contagem, Ibirité, Lagoa Santa, Nova Lima, Pedro Leopoldo, Raposos, Ribeirão das Neves, Rio Acima, Sabará, Santa Luzia e Vespasiano. Quase duas décadas após sua institucionalização, à RMBH foram acrescidos os municípios de Mateus Leme, Igarapé, Esmeraldas e Brumadinho, por meio da Constituição Estadual de 1989. Em decorrência de novas emancipações também foram incorporados Juatuba e São José da Lapa, desmembrados em 1992 de Mateus Leme e Vespasiano, respectivamente. Em 1999, dois outros municípios foram adicionados: Confins e Mário Campos (emancipados de Lagoa Santa e Ibirité, nessa ordem). Nesse mesmo ano,

\footnotetext{
${ }^{4}$ Souza e Brito (2008) analisaram o processo de expansão urbana de Belo Horizonte e da Região Metropolitana de Belo Horizonte (RMBH) em direção ao município de Ribeirão das Neves. Tendo como fonte de dados os Censos Demográficos de 1991 e 2000 e as pesquisas origem-destino de 1992 e 2002, os resultados apresentados indicaram que essa expansão urbana em direção a Ribeirão das Neves não se relaciona ao dinamismo econômico do município, mas sim a um processo de exclusão de Belo Horizonte. Isso devido ao fato de que Ribeirão das Neves atrai um grande contingente populacional, principalmente por meio da dinâmica de seu mercado imobiliário, caracterizado por alto grau de informalidade. Nesse trabalho, com base na OD (2002), esses autores observaram que $70 \%$ dos trabalhadores de Ribeirão das Neves moraram anteriormente em Belo Horizonte, sendo que $30 \%$ trabalhavam informalmente na capital mineira.
} 
os municípios de Florestal e Rio Manso, que integravam o colar metropolitano, também foram incorporados à RM. Os municípios de São Joaquim de Bicas e Sarzedo, desmembrados de Igarapé e Ibirité, respectivamente, também passaram a fazer parte da RM. De 2000 a 2002, a RMBH sofreu nova alteração. Os municípios de Jaboticatubas e Taquaraçu de Minas, que integravam o colar metropolitano, passaram a fazer parte da RMBH. Nesse mesmo período, Itabirito saiu da RM e voltou a pertencer ao colar. Em 2002, Itatiaiuçu saiu do colar e passou para a RMBH, completando o número atual de 34 municípios, como observado na Figura 1. Algumas dessas incorporações à RMBH foram motivadas por questões meramente políticas, a exemplo do caso de Itabirito, incluído em 1999, como descreve Gouvêa (2005). Esse mesmo autor também destaca a incorporação de municípios que não atendiam a critérios básicos no campo do urbanismo e que, na realidade, existe uma região metropolitana "real” dentro da RMBH “legal” (GOUVÊA, 2005). ${ }^{5}$

FIGURA 1

Formação territorial da Região Metropolitana de Belo Horizonte/MG

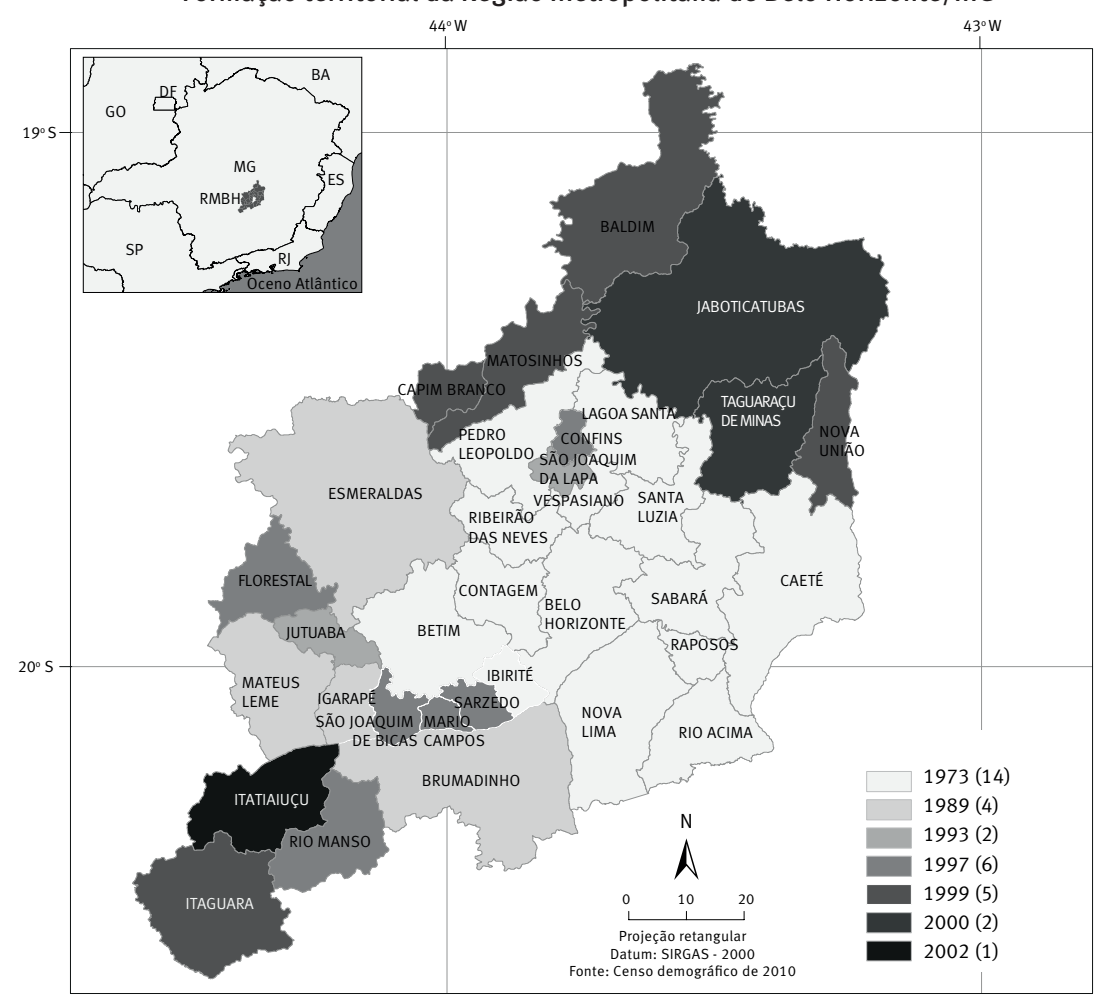

Fonte: Base de dados digital do IBGE, Censo Demográfico 2010. Elaboração dos autores.

\footnotetext{
${ }^{5}$ Cabe destacar o caso do município de Sete Lagoas, localizado na porção noroeste da RMBH. Embora não incluído na Região Metropolitana de Belo Horizonte, os fluxos migratórios e pendulares com destino à capital mineira foram investigados por Nogueira, Garcia e Moreira (2010). Nesse estudo, os autores concluíram que, na análise dos fluxos populacionais de Sete Lagoas com o núcleo metropolitano, as articulações e interações eram de extrema relevância, reafirmando-se, assim, a polarização exercida pela metrópole mineira sobre a cidade. Tais afirmações corroboram as reflexões de 0jima (2010) e Gouvêa (2005) acerca da existência e manutenção de "alta pendularidade fora do contexto metropolitano" na RMBH, o que pode ser verificada, inclusive, para o caso de Sete Lagoas.
} 
Utilizando os microdados amostrais do Censo Demográfico de 2010, pela identificação do município de residência, de trabalho e de frequência à escola (ou creche), ${ }^{6}$ estimaram-se os volumes e vetores dos ditos movimentos pendulares. Obteve-se, dessa forma, uma matriz de origem-destino dos deslocamentos pendulares diários, o que permitiu identificar não apenas os fluxos que envolviam o núcleo metropolitano, mas também aqueles que englobavam todos os demais municípios de cada região. Com essa matriz foi possível elaborar um conjunto de indicadores, incluindo um indicador sintético agregado, denominado Índice de Integração Regional (IIR).

O IIR foi derivado da combinação de três outros indicadores, denominados razão de pendularidade interna (RPI), razão de conectividade pendular (RCP) e razão de pendularidade nuclear (RPN), obtidos da seguinte forma:

$\mathrm{RPI}=\sum_{1}^{n} \frac{M P i_{i j}}{M P e_{i j}}$

Onde:

$M P i_{i j}=$ movimentos pendulares envolvendo município $i$ e $j$, ambos pertencentes à RM (deslocamentos internos);

$M P e_{i j}=$ movimentos pendulares com origem no município $i$ (pertencente à RM) e destino no município $j$ não pertencente à RM (deslocamentos para fora da RM).

$\mathrm{RCP}=\sum_{1}^{n} \frac{C P e_{i j}}{C P p_{i j}}$

Onde:

$C P e_{i j}=$ número de conexões efetivas do município $i$ na região $j$ (conexões municipais efetivas internas);

$C P p_{i j}=$ número de conexões possíveis do município $i$ na região $j$ (total de municípios menos um).

$\mathrm{RPN}=\sum_{1}^{n} \frac{M \operatorname{Pn}_{i j}}{\operatorname{Ptem}_{i j}}$

Onde:

$M P n_{i j}=$ movimentos pendulares envolvendo município $i$ para o núcleo metropolitano na região $j$ (deslocamentos internos com destino ao NM);

Ptem $_{i j}=$ população que trabalha ou estuda no município $i$ na região $j$.

\footnotetext{
${ }^{6}$ Esses últimos foram combinados, o que permitiu analisar os deslocamentos com a dupla finalidade: trabalho ou estudo. Destaca-se que, no Censo 2010, o IBGE desagregou as informações referentes ao município de trabalho e estudo. Nos casos em que foi identificado que a pessoa pesquisada reside em um município, trabalha em outro e estuda em um terceiro, nesse estudo, considerou-se somente o município de trabalho.
} 
Esses coeficientes representam diferentes dimensões do nível de integração municipal, considerando a mobilidade pendular proxi da mobilidade metropolitana (RPI), da conexão intermunicipal (RCP) e da centralidade exercida pelo NM (RPN). De cada um desses coeficientes (RPI, RPC e RPN) foi padronizado em um índice, obtido pela razão entre diferença entre o enésimo valor e o mínimo observado e a diferença entre os valores máximo e mínimo de cada indicador. Como resultado têm-se os IPI, ICP e IPN, cujos escores foram convertidos na escala de 0 a 1 ( 0 para o valor mais baixo no indicador e 1 para o mais elevado), conforme descrito nas seguintes expressões:

$$
\begin{aligned}
& I P I=\frac{R P I_{i j}-\text { RPImin }_{i j}}{\text { RPImax }_{i j}-\text { RPImin }_{i j}} \\
& I C P=\frac{R P C_{i j}-R P C \min _{i j}}{R P C \max _{i j}-R P C \min _{i j}} \\
& I P N=\frac{R P N_{i j}-R P N \min _{i j}}{\text { RPNmax }_{i j}-\text { RPNmin }_{i j}}
\end{aligned}
$$

Onde:

$R P I_{i j} ; R P C_{i j} ; R P N_{i j}=$ razão do indicador $w$, do município $i$ na região $j$; $R P I m i n i j ;$; $P C \min _{i j} ; R P N \min _{i j}=$ razão mínima do indicador $w$, para o município $i$ na região $j ;$ $R P \operatorname{Imax}_{i j} ; R P C \max _{i j} ; R P N \max _{i j}=$ razão máxima do indicador $w$, para o município $i$ na região $j$;

Os resultados, para fins de análise, utilizando a plataforma $\operatorname{ArcMap}{ }^{\circledR}$ do sistema ArcGis ${ }^{\circledR}$, foram agrupados em cinco classes pelo método denominado "quebra natural”, também conhecido como distribuição de Jenks (Natural Break ou Natural Break Points). ${ }^{7}$ Os valores referentes aos respectivos IIRs foram representados em cartogramas, cujas classes foram denominadas: "muito baixa”, "baixa”, “média”, "média alta” e "alta”, conforme representado na Figura 2. Para fins de comparação, os resultados do ICM foram contrapostos àqueles apresentados nas propostas metodológicas de Ojima (2011) e do IBGE (2015). ${ }^{8}$

\footnotetext{
7 o método da quebra natural de Jenks (1967) tem como objetivo encontrar os intervalos de classes com o propósito de minimizar a variância dentro das classes. Assim, procura-se por agrupamentos que ocorram "naturalmente" nos dados, buscando uma homogeneidade interna para cada classe. Portanto, por meio dessa metodologia, o mapa de probabilidade pode ser interpretado como um mapa que foi divido em cinco classes, de modo que essas classes possuem probabilidades estimadas semelhantes (ALBUQUERQUE, 2008).

8 O trabalho de Ojima (2011) utilizou como base o conceito de Aglomeração Urbana (AU), como definido na pesquisa da Rede Urbana Brasileira, publicada pelo Ipea, IBGE e Unicamp (2000). 0 autor utiliza uma metodologia de análise das AUs brasileiras com base em dados censitários que identificam os movimentos pendulares, de forma a incorporar ou não os municípios que efetivamente possuem uma integração demográfica. Ojima se baseou nos critérios de origem da pendularidade. A linha de corte foi $10,5 \%$ da população de pelo menos uma área de ponderação realizando movimentos pendulares. No destino, o autor utilizou a seguinte linha de corte: os movimentos pendulares deveriam representar pelo menos $5 \%$ da população do município de destino. Também foi incorporado um critério adicional de contiguidade para os casos em que algum município da RM não apresentasse contiguidade física entre as divisões político-administrativas dos municípios integrantes ao conjunto principal de municípios da Aglomeração Urbana. Nesses casos, foram considerados os municípios intermediários. Já o estudo de “Arranjos Populacionais e Concentrações Urbanas do Brasil”, realizado pelo Instituto Brasileiro de Geografia e Estatística (IBGE, 2015), com base nos dados extraídos do Censo Demográfico de 2010, considerou arranjo populacional o agrupamento de dois ou mais municípios onde há uma forte integração populacional, devido aos movimentos pendulares para trabalho e/ou estudo, ou dada a contiguidade entre as manchas urbanizadas principais.
} 


\section{A mobilidade pendular na RMBH: os padrões e os níveis de integração regional}

Em uma análise preliminar, conforme dados apresentados na Tabela 1, nota-se que a pendularidade por motivos de trabalho e/ou estudo envolveu um total de 538.391 pessoas residentes em municípios da RMBH que se deslocaram regularmente para trabalhar e/ou estudar dentro e fora da RM em 2010. Esse contingente pendular correspondia a aproximadamente $11 \%$ do total da população da região, o que, a priori, indica a elevada relevância da mobilidade populacional na dinâmica demográfica metropolitana. Desse total, 511.172 casos referem-se a movimentos com origem e destino nos municípios metropolitanos (94,94\% do total regional). Alguns municípios, em decorrência do próprio tamanho populacional, apresentam um volume de deslocamento pendular muito elevado, como Contagem (112.432), Ribeirão das Neves (89.188), Betim (53.152), Ibirité (50.635) e Santa Luzia (50.541). Em outros municípios, com números bem mais acanhados de população residente, como Itatiaiuçu (142) e Itaguara (178), a mobilidade pendular apresenta baixa expressão.

Contudo, quando analisada a razão entre os deslocamentos internos e externos (aqueles com destino a municípios que não integram a RMBH), algumas particularidades locais tornam-se notáveis. Em Itatiaiuçu, Itaguara e Baldim, os fluxos referentes à pendularidade metropolitana apresentam razão inferior a 0,1 , o que significa, nesses casos, que, para cada pessoa que se desloca para trabalho e estudo na RM, pelo menos outras nove tinham como destino um município fora dos limites metropolitano. A baixa conectividade metropolitana desses três municípios também é observada quando analisados os vetores de ligações pendulares e o peso das interações com o núcleo. Considerando essas duas variáveis, também merece destaque os casos de Nova União, São Joaquim de Bicas e Taquaraçu de Minas (todos com baixos níveis em ambas). Nesses três municípios foram observados baixos níveis de conexão pelos vetores de ligação pendular com, respectivamente, apenas 6, 14 e 9 outros municípios, envolvendo um total de 205, 636 e 123 pessoas, nessa ordem, que se deslocavam diariamente para trabalho ou estudo em Belo Horizonte.

Os resultados referentes ao IPI, ICP e IPN, representados na Figura 2, demonstram que, para além de uma esperada relação direta de proximidade/vizinhança com o NM, dada a prevalência de maior integração com os municípios limítrofes, há diferenciações regionais notáveis. Se os padrões espaciais referentes ao IPI e IPN são similares, indicando maiores valores nos municípios do vetor norte/noroeste, que incluem Ribeirão das Neves, Santa Luzia, Vespasiano e Sabará, a distribuição do ICP sugere maior relevância na porção oeste, que englobam Contagem e Betim. Esses dois municípios apresentam elevado nível de ligação com os demais municípios metropolitanos, especialmente pela capacidade de atração de força de trabalho nas atividades industrial e de serviços. Ainda que seja expressiva a relação com a própria capital do estado, as interações extrapolam uma suposta dependência do NM. Deve-se novamente mencionar os casos de Itatiaiuçu, Itaguara e Baldim, que exibiam os menores níveis nas três classificações estabelecidas. A análise do IIR reforça 
a maior mobilidade envolvida no eixo norte/noroeste. Cabe, contudo, destacar o caso de Ibirité. Trata-se de um município em uma importante zona de expansão do tecido urbano no eixo sul, com forte nível de interação com a regional Barreiro (região administrativa ao sul de Belo Horizonte). No outro extremo, além dos municípios de Itatiaiuçu, Itaguara e Baldim, que confirmaram sua baixa integração regional, importa ressaltar os casos de Rio Manso (porção sul) e Forestal (oeste). Trata-se de municípios de pequeno porte demográfico e baixo nível de interação metropolitana pela mobilidade pendular.

TABELA 1

Indicadores de pendularidade (mobilidade, ligações e interações) Municípios da Região Metropolitana de Belo Horizonte - 2010

\begin{tabular}{|c|c|c|c|c|c|c|c|c|c|}
\hline \multirow[t]{2}{*}{ Municípios } & \multicolumn{3}{|c|}{$\begin{array}{c}\text { Mobilidade } \\
\text { (fluxo de pessoas) }\end{array}$} & \multicolumn{3}{|c|}{$\begin{array}{c}\text { Ligações } \\
\text { (entre municípios da RM) }\end{array}$} & \multicolumn{3}{|c|}{$\begin{array}{c}\text { Interaçãa com o NM } \\
\text { (que trabalham/estudam no) }\end{array}$} \\
\hline & $\begin{array}{l}\text { Interna } \\
\text { (A) }\end{array}$ & $\begin{array}{l}\text { Externa } \\
\text { (B) }\end{array}$ & $A / B$ & $\begin{array}{l}\text { Efetivas } \\
\text { (A) }\end{array}$ & $\begin{array}{l}\text { Possíveis } \\
\text { (B) }\end{array}$ & $A / B$ & $\begin{array}{l}\text { NM } \\
(\mathrm{A})\end{array}$ & $\begin{array}{l}\text { Mun. de } \\
\text { residencia } \\
\text { (B) }\end{array}$ & $A / B$ \\
\hline Baldim & 178 & 217 & 0,82 & 7 & 33 & 0,21 & 124 & 4.718 & 0,03 \\
\hline Betim & 53.152 & 3.531 & 15,05 & 27 & 33 & 0,82 & 28.180 & 224.273 & 0,13 \\
\hline Brumadinho & 3.103 & 341 & 9,09 & 17 & 33 & 0,52 & 2.134 & 21.568 & 0,10 \\
\hline Caeté & 4.397 & 804 & 5,47 & 15 & 33 & 0,45 & 2.874 & 21.964 & 0,13 \\
\hline Capim Branco & 1.751 & 190 & 9,22 & 10 & 33 & 0,30 & 250 & 4.220 & 0,06 \\
\hline Confins & 906 & 57 & 15,77 & 9 & 33 & 0,27 & 238 & 3.732 & 0,06 \\
\hline Contagem & 112.432 & 7.672 & 14,65 & 25 & 33 & 0,76 & 88.772 & 335.852 & 0,26 \\
\hline Esmeraldas & 12.920 & 419 & 30,84 & 15 & 33 & 0,45 & 6.109 & 28.609 & 0,21 \\
\hline Florestal & 508 & 256 & 1,98 & 7 & 33 & 0,21 & 181 & 4.352 & 0,04 \\
\hline Ibirité & 50.635 & 1.396 & 36,26 & 18 & 33 & 0,55 & 35.378 & 63.135 & 0,56 \\
\hline Igarapé & 3.928 & 315 & 12,45 & 13 & 33 & 0,39 & 728 & 20.157 & 0,04 \\
\hline Itaguara & 264 & 383 & 0,69 & 4 & 33 & 0,12 & 193 & 8.301 & 0,02 \\
\hline Itatiaiuçu & 142 & 447 & 0,32 & 7 & 33 & 0,21 & 54 & 6.262 & 0,01 \\
\hline Jaboticatubas & 1.034 & 207 & 4,99 & 10 & 33 & 0,30 & 636 & 10.679 & 0,06 \\
\hline Juatuba & 2.595 & 198 & 13,12 & 9 & 33 & 0,27 & 673 & 12.778 & 0,05 \\
\hline Lagoa Santa & 6.780 & 381 & 17,77 & 14 & 33 & 0,42 & 4.530 & 32.990 & 0,14 \\
\hline Mário Campos & 3.013 & 87 & 34,47 & 15 & 33 & 0,45 & 1.260 & 6.269 & 0,20 \\
\hline Mateus Leme & 2.078 & 821 & 2,53 & 12 & 33 & 0,36 & 732 & 16.450 & 0,04 \\
\hline Matozinhos & 3.131 & 886 & 3,53 & 18 & 33 & 0,55 & 1.073 & 20.615 & 0,05 \\
\hline Nova Lima & 15.314 & 1.144 & 13,38 & 14 & 33 & 0,42 & 13.475 & 46.330 & 0,29 \\
\hline Nova União & 387 & 54 & 7,12 & 6 & 33 & 0,18 & 205 & 3.317 & 0,06 \\
\hline Pedro Leopoldo & 6.792 & 584 & 11,63 & 16 & 33 & 0,48 & 3.139 & 34.499 & 0,09 \\
\hline Raposos & 4.569 & 106 & 43,24 & 9 & 33 & 0,27 & 2.526 & 5.616 & 0,45 \\
\hline Ribeirão das Neves & 89.188 & 2.945 & 30,28 & 25 & 33 & 0,76 & 76.322 & 126.324 & 0,60 \\
\hline Rio Acima & 1.328 & 93 & 14,28 & 12 & 33 & 0,36 & 829 & 5.043 & 0,16 \\
\hline Rio Manso & 326 & 100 & 3,27 & 9 & 33 & 0,27 & 97 & 3.499 & 0,03 \\
\hline Sabará & 38.737 & 1.015 & 38,15 & 17 & 33 & 0,52 & 35.970 & 52.943 & 0,68 \\
\hline Santa Luzia & 50.541 & 1.365 & 37,02 & 20 & 33 & 0,61 & 45.549 & 94.362 & 0,48 \\
\hline São Joaquim de Bicas & 3.225 & 167 & 19,33 & 14 & 33 & 0,42 & 636 & 13.105 & 0,05 \\
\hline São José da Lapa & 4.450 & 112 & 39,57 & 14 & 33 & 0,42 & 2.843 & 10.181 & 0,28 \\
\hline Sarzedo & 6.174 & 237 & 26,09 & 13 & 33 & 0,39 & 2.988 & 12.489 & 0,24 \\
\hline Taquaraçu de Minas & 242 & 17 & 14,50 & 9 & 33 & 0,27 & 123 & 2.572 & 0,05 \\
\hline Vespasiano & 26.951 & 668 & 40,34 & 18 & 33 & 0,55 & 23.308 & 48.294 & 0,48 \\
\hline
\end{tabular}

Fonte: Censo Demográfico de 2010 (dados da amostra). 


\section{FIGURA 2}

Índice de Pendularidade Interna (IPI), Índice de Conectividade Pendular (ICP) e Índice de Pendularidade Nuclear (IPN)

Municípios da Região Metropolitana de Belo Horizonte - 2010
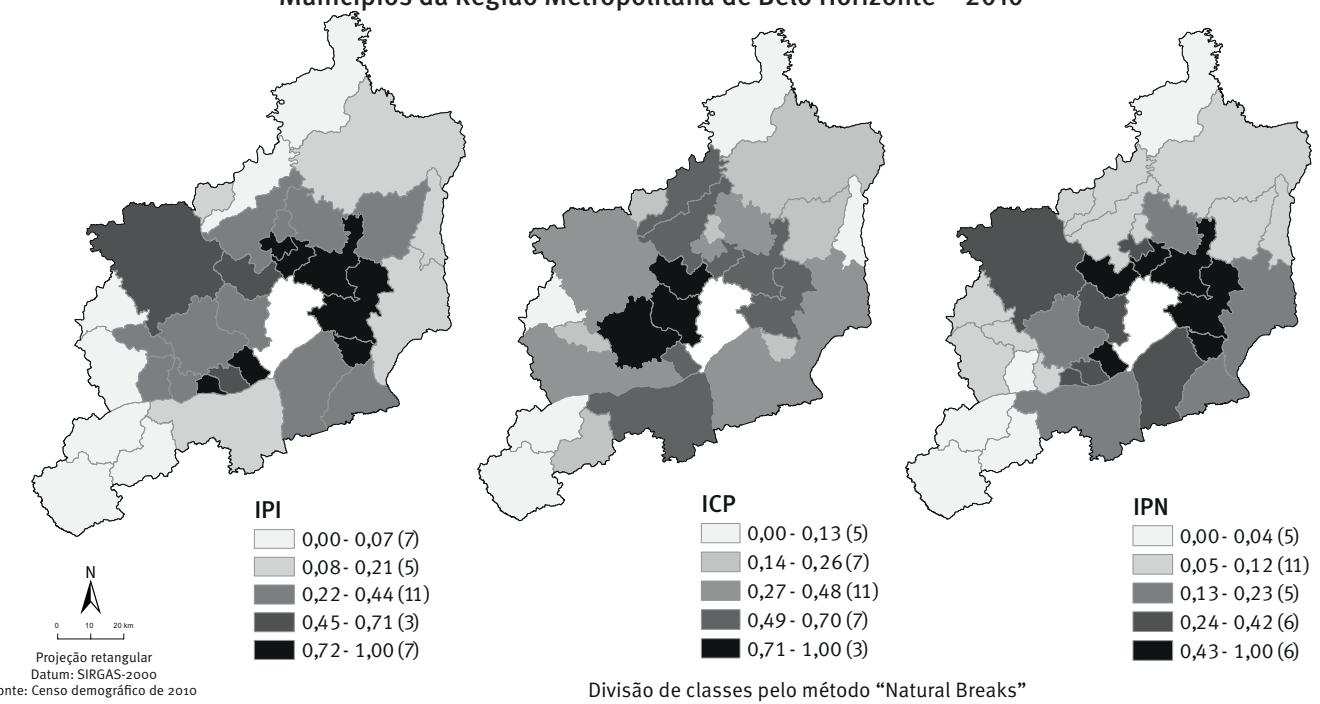

Fonte: Base de dados digital do IBGE - Censo Demográfico 2010. Elaboração dos autores.

FIGURA 3

Índice de Integração Regional (IIR)

Municípios da Região Metropolitana de Belo Horizonte - 2010

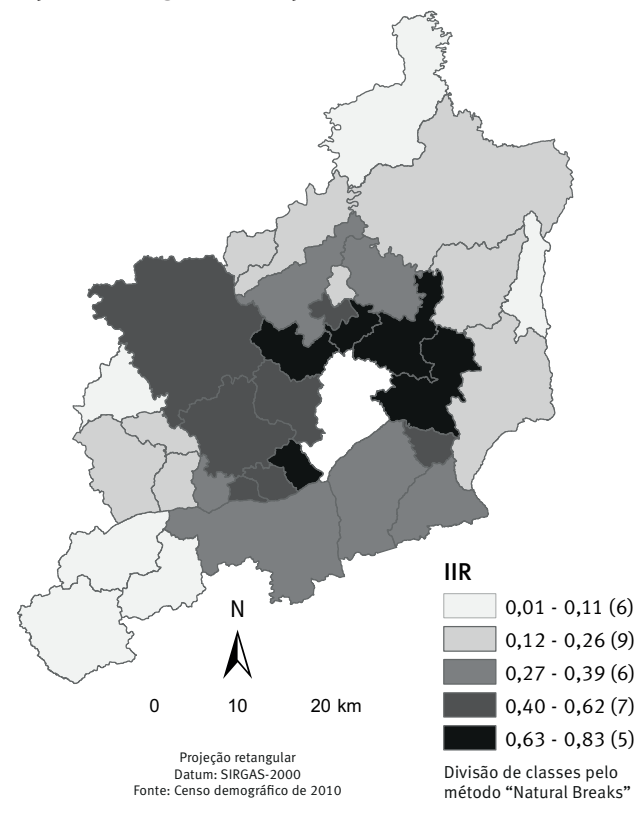

Fonte: Base de dados digital do IBGE - Censo Demográfico 2010. Elaboração dos autores. 
O Quadro 1 permite comparar os indicadores apresentados nesse trabalho aos resultados propostos por Ojima (2011) e pelo IBGE (2015). Se considerarmos os casos de concordância entre os três estudos, não seriam enquadrados no recorte regional metropolitano os municípios de Baldim, Florestal, Itaguara, Itatiaiuçu, Jaboticatubas, Mateus Leme, Nova União, Rio Manso e Taquaraçu de Minas. Desses nove municípios, apenas Mateus Leme e Taquaraçu de Minas não foram classificados com integração "muito baixa" (os dois casos foram incluídos na classificação "baixa”). Resultados similares aos expostos nessa proposta foram apresentados por Ojima (2011), ao indicar que a RMBH seria composta por apenas 25 municípios. 0 autor utilizou os limites dos fluxos pendulares relativos às viagens de origem da pendularidade (com 10,5\% da população de pelo menos uma área de ponderação realizando movimentos pendulares) e de destino da pendularidade que representam pelo menos $5 \%$ da população do município de destino. De acordo com o IBGE (2015), com diferenças pontuais em relação ao trabalho anterior, a RMBH seria composta por 23 municípios, sendo que apenas 15 apresentam mancha urbana contígua, sendo eles: Belo Horizonte, Betim, Confins, Contagem, Ibirité, Igarapé, Lagoa Santa, Mário Campos, Nova Lima, Pedro Leopoldo, Sabará, Santa Luzia, São Joaquim de Bicas, São José da Lapa, Sarzedo e Vespasiano. Segundo os mesmos critérios apresentados pelo IBGE (2015), os municípios de Baldim, Florestal, Itaguara, Jaboticatubas, Juatuba, Mateus Leme, Rio Manso e Taquaraçu de Minas não participam de nenhum arranjo metropolitano regional.

Ainda que a interpretação dos resultados requeira cuidados em relação aos limites decorrentes do real significado da mobilidade pendular como indicador de integração regional, bem como da natureza de recorte temporal transversal (cross-section), há certamente a necessidade de avaliação da abrangência da suposta dispersão urbana, inclusive para fins de planejamento e gestão territorial do espaço metropolitano. Se as consequências sociais do processo de dispersão urbana ainda não convergiram em um consenso teórico, as evidências empíricas indicam níveis consideravelmente distintos de integração regional na RMBH. Além da área geográfica efetivamente conurbada do tecido metropolitano, especialmente dos municípios localizados nas porções oeste e norte da capital mineira, há núcleos urbanos mais dispersos que são bem integrados regionalmente, embora os limites metropolitanos também incluam áreas de baixa conexão regional, que não se enquadram nos modelos clássicos descritos na literatura. 
QUADRO 1

Indicação e nível de integração metropolitana

Municípios da Região Metropolitana de Belo Horizonte - 2010

\begin{tabular}{|c|c|c|c|}
\hline Municípios & $\begin{array}{l}\text { Ojima } \\
\text { (2011) }\end{array}$ & $\begin{array}{l}\text { IBGE } \\
(2015)\end{array}$ & $\begin{array}{c}\text { ICM } \\
\text { (Nível) }\end{array}$ \\
\hline Baldim & & & Muito baixo \\
\hline Betim & $x$ & $x$ & Alto \\
\hline Brumadinho & $x$ & $x$ & Médio \\
\hline Caeté & $x$ & $x$ & Baixo \\
\hline Capim Branco & $x$ & & Baixo \\
\hline Confins & $x$ & $x$ & Baixo \\
\hline Contagem & $x$ & $x$ & Alto \\
\hline Esmeraldas & $x$ & $x$ & Alto \\
\hline Florestal & & & Muito baixo \\
\hline Ibirité & $x$ & $x$ & Muito alto \\
\hline Igarapé & $x$ & $x$ & Baixo \\
\hline Itaguara & & & Muito baixo \\
\hline Itatiaiuçu & & & Muito baixo \\
\hline Jaboticatubas & & & Baixo \\
\hline Juatuba & $x$ & & Baixo \\
\hline Lagoa Santa & $x$ & $x$ & Médio \\
\hline Mário Campos & $x$ & $x$ & Alto \\
\hline Mateus Leme & & & Baixo \\
\hline Matozinhos & $x$ & & Baixo \\
\hline Nova Lima & $x$ & $x$ & Médio \\
\hline Nova União & & & Muito baixo \\
\hline Pedro Leopoldo & $x$ & $x$ & Médio \\
\hline Raposos & $x$ & $x$ & Alto \\
\hline Ribeirão das Neves & $x$ & $x$ & Muito alto \\
\hline Rio Acima & $x$ & $x$ & Médio \\
\hline Rio Manso & & & Muito baixo \\
\hline Sabará & $x$ & $x$ & Muito alto \\
\hline Santa Luzia & $x$ & $x$ & Muito alto \\
\hline São Joaquim de Bicas & $x$ & $x$ & Médio \\
\hline São José da Lapa & $x$ & $x$ & Alto \\
\hline Sarzedo & $x$ & $x$ & Alto \\
\hline Taquaraçu de Minas & & & Baixo \\
\hline Vespasiano & $x$ & $x$ & Muito alto \\
\hline
\end{tabular}

Fonte: IBGE (2015); OJIMA (2011).

Nota: Em destaque os municípios que não atendiam, simultaneamente, aos critérios definidos nos três trabalhos considerados.

\section{Considerações finais}

Não obstante as naturais incertezas e controvérsias que envolvem a análise da dinâmica econômica e social da população em determinada região, tornam-se cada vez mais necessários novos investimentos em pesquisas que avaliem o significado e os possíveis impactos dos movimentos espaciais da população no espaço metropolitano, seja pela migração, seja 
mesmo pela dita mobilidade pendular. A Região Metropolitana de Belo Horizonte, ainda que apresente condições comuns à boa parte das RMs do país, compreende um espaço especialmente peculiar. Na metade do século passado, a capital do estado tinha pouco mais de 350 mil habitantes. Em menos de 50 anos Belo Horizonte apresentou um expressivo crescimento demográfico, seguido de um rápido arrefecimento, resultado tanto da queda das taxas de fecundidade como da inversão no saldo migratório. Especialmente a partir de década de 1980, vários municípios da periferia metropolitana apresentaram níveis de crescimento populacional significativamente superior ao núcleo. Se os sinais de dispersão espacial da população residente são insuficientes para caracterizar um quadro típico de desconcentração espacial, como proposto em modelos clássicos da economia regional, parece haver outros sinais de ganho de autonomia econômica em diversos espaços além do município core, como os dados de mobilidade pendular parecem indicar.

Os resultados apresentados nesse trabalho podem ser sintetizados em três conclusões gerais:

- para além da esperada relação entre integração metropolitana e proximidade em relação a Belo Horizonte, os diferentes padrões espaciais indicam uma associação com as características regionais, especialmente aquelas decorrentes das atividades e condições econômicas locais;

- alguns municípios, como Baldim, Florestal, Itaguara, Itatiaiuçu, Jaboticatubas, Nova União e Rio Manso, que atualmente integram a RMBH, apresentam baixíssimo nível de integração regional, extrapolando os reais limites do que poderia ser definido como espaço metropolitano;

- há municípios em que a interação metropolitana vai além de uma simples ação de polarização do núcleo, o que sugere a difusão de novas centralidades regionais, a exemplo do vetor oeste (Contagem e Betim).

Essa controvérsia requer, a priori, como indicado por Ojima (2016), uma reflexão sobre o próprio recorte metropolitano. 0 autor indica duas opções: predefinir o limite territorial (município A, região metropolitana B, etc.) e buscar analisar a dispersão urbana e os deslocamentos populacionais dentro desse recorte regional; ou estabelecer, com base nos deslocamentos populacionais, a extensão do território que deverá ser considerado o recorte de estudo. Isso significa, por exemplo, levar em conta que a dispersão urbana não deve ser entendida apenas como a expansão da mancha urbanizada, mas deve ser repensada para que se compreendam os processos de ocupação e a sua forma. Para Araújo e Holzer (2016), o fenômeno do espraiamento metropolitano e sua dinâmica não se restringem a variáveis demográficas e à urbanização contínua. Aspectos como mudanças implícitas no processo de organização socioespacial e os mecanismos de ação e seus agentes e a forma como impactam a formação de uma nova paisagem precisam ser investigados. Ainda que a interpretação dos resultados apresentados deva ser contraposta às especificidades do caso e do recorte temporal, bem como aos limites do significado dos movimentos pendulares 
como proxi da integração regional metropolitana, parece razoável a necessidade de uma análise mais detalhada e acurada do que é chamado genericamente de região metropolitana no país. A reflexão não deve se ater de forma restrita à redefinição da configuração territorial e à atualização do recorte regional, mas precisa incluir as características e a dinâmica que configuram novas espacialidades que se consolidam em torno das principais metrópoles do país. Poderiam ser denominadas de áreas de transbordo da polarização metropolitanas? Ou definiriam novos polos regionais de influência metropolitana? Essas, entre outras, são questões que não devem ser ignoradas.

\section{Referências}

ARAÚJO, E. C. de; HOLZER, W. Dispersão urbana e planejamento urbano regional: inquietações. In: OJIMA, R.; MARANDOLA JR., E. Dispersão urbana e mobilidade populacional. São Paulo: Blucher, 2016.

ALBUQUERQUE, P. H. M. Conglomerados espaciais: uma nova proposta. 2008. 117 f. Dissertação (Mestrado em Estatística) - Departamento de Estatística, Instituto de Ciências Exatas, Universidade Federal de Minas Gerais, Belo Horizonte, 2008.

CASTELLO BRANCO, M. L. G. Espaços urbanos: uma proposta para o Brasil. Rio de Janeiro: IBGE, 2003.

CASTELLO BRANCO, M. L. G.; FIRKOWSKI, O. L. F. C.; MOURA, R. Movimento pendular: abordagem teórica e reflexões sobre o uso do indicador. In: ENCONTRO NACIONAL DA ANPUR, 11. 2005, Salvador. Anais... Salvador: Anpur, maio 2005.

CREA-MG - Conselho Regional de Engenharia e Agronomia de Minas Gerais. Mobilidade: Região Metropolitana de Belo Horizonte. Belo Horizonte: Crea-MG, 2014.

CRUZ, M. F. Condicionantes metropolitanos para políticas públicas: análise dos transportes coletivos na Região Metropolitana de São Paulo (1999-2009). 2010. 146 f. Dissertação (Mestrado em Administração Pública e Governo) - Escola de Administração de Empresas de São Paulo da Fundação Getúlio Vargas, São Paulo, 2010.

DESCHAMPS, M.; DELGADO, P.; MOURA, R.; CASTELLO BRANCO, M. L. Nível de integração dos municípios à dinâmica metropolitana. Curitiba: Ipardes, 2007.

DURANTON, G. Delineating metropolitan areas: measuring spatial labour market networks through commuting patterns. Processed, Pennsylvania, 2013. Disponivel em: 〈http://real. wharton.upenn.edu/ duranton/Duranton_Papers/Current_Research/MSA_Colombia.pdf . Acesso em: 04 jan. 2015.

FREY, W. H.; SPEARE, JR. A. Metropolitan areas as functional communities: a proposal for a new definition. Research Report, Population Studies Center, University of Michigan USA, July 1992.

GOUVÊA, R. G. A questão metropolitana no Brasil. 1. ed. Rio de Janeiro: FGV, 2005.

IBGE - Instituto Brasileiro de Geografia e Estatística. Arranjos populacionais e concentrações urbanas no Brasil. Rio de Janeiro: IBGE, 2015.

Censo demográfico 2010. Rio de Janeiro: IBGE, 2010.

IPEA; IBGE; UNICAMP/IE/NESUR. Configurações atuais e tendências das redes urbanas. Brasília: Ipea, IBGE, Unicamp, 2002 (Série Caracterização e Tendências da Rede Urbana do Brasil, n. 1). 
JARDIM, A. de P. Reflexões sobre a mobilidade pendular. In: OLIVEIRA, L. A. P. de; OLIVEIRA, A. T. R. de. Reflexões sobre os deslocamentos populacionais no Brasil. Rio de Janeiro: IBGE, 2011.

JARDIM, A. de P.; ERVATTI, L. R. Migração pendular intrametropolitana no Rio de Janeiro: reflexões sobre o seu estudo a partir dos Censos Demográficos de 1980 e 2000. Rio de Janeiro: IBGE, 2007.

LIMONAD, E. Urbanização dispersa mais uma forma de expressão urbana? Revista Formação, v. 14, n. 1, p. 31-45, 2011.

LOBO, C. Dispersão espacial da população no Brasil. Mercator, v. 15, n. 3, p. 19-36, 2016.

Mobilidade pendular e a dispersão espacial da população: evidências com base nos fluxos com destino às principais metrópoles brasileiras. Caderno de Geografia, v. 26, n. 45, p. 285-298, 2016.

LOBO, C.; MATOS, R.; CARDOSO, L.; COMINI, L.; PINTO, G. Expanded commuting in the metropolitan region of Belo Horizonte: evidence for reverse commuting. Revista Brasileira de Estudos de População, v. 32, n. 2, p. 219-233, 2015.

LOBO, C.; CARDOSO, L.; MATOS, R. Mobilidade pendular e centralidade espacial: considerações sobre o caso da Região Metropolitana de Belo Horizonte. In: XXIII ANPET - Congresso de Pesquisa e Ensino em Transportes. Anais... Vitória/ES, 2009.

MOURA, R.; BRANCO, M. L. G. C.; IRKOWSKI, O. L. C. de F. Movimento pendular e perspectivas de pesquisas em aglomerados urbanos. São Paulo em Perspectiva, v. 19, n. 4, p. 121-133, 2005.

NOGUEIRA, M.; GARCIA, R. A.; MOREIRA, K. C. A importância dos fluxos populacionais para compreensão da centralidade urbana: o caso de Sete Lagoas /MG. In: CONGRESSO BRASILEIRO DE ORGANIZAÇÃO DO ESPAÇO, X SEMINÁRIO DE PÓS-GRADUAÇÃO EM GEOGRAFIA DA UNESP. Anais... Rio Claro: Unesp, outubro de 2010.

OJIMA, R. Pessoas, prédios e ruas: por uma perspectiva demográfica dos processos urbanos contemporâneos. In: OJIMA, R.; MARANDOLA JR., E. Dispersão urbana e mobilidade populacional. São Paulo: Blucher, 2016.

Fronteiras metropolitanas: um olhar a partir dos movimentos pendulares. Revista Paranaense de Desenvolvimento, n. 121, p. 115-132, jul./dez. 2011.

OJIMA, R.; MARANDOLA JR., E.; PEREIRA, R. H. M.; DA SILVA, R. B. O estigma de morar longe da cidade: repensando o consenso sobre as "cidades-dormitório" no Brasil. Cadernos Metrópole, v. 12, n. 24, p. 395-415, 2010.

OJIMA, R.; SILVA, R. B.; PEREIRA, R. H. M. A mobilidade pendular na definição das cidades-dormitório: caracterização sociodemográfica e novas territorialidades no contexto da urbanização brasileira. Cadernos IPPUR, v. 21, n. 2, p. 111-132, ago./dez. 2007.

PEREIRA, R. H. M.; HERRERO, V. Mobilidade pendular: uma proposta teórico-metodológica. Rio de Janeiro: Ipea, mar. 2009 (Texto para Discussão, n. 1.395). Disponível em: hhttp://goo.gl/ Kz6f2Z>. Acesso em: 20 abr. 2014.

REIS, N. G. Notas sobre urbanização dispersa e novas formas de tecido urbano. São Paulo: Via das Artes, 2006.

SOUZA, J. de; BRITO, F. Expansão urbana de Belo Horizonte e da RMBH: a mobilidade residencial e o processo de periferização, nos anos 80 e 90. In: XIII SEMINÁRIO SOBRE A ECONOMIA MINEIRA. Anais... Belo Horizonte: Cedeplar, Universidade Federal de Minas Gerais, 2008.

TASCHNER, S. P.; BÓGUS, L. M. M. Mobilidade espacial da população brasileira: aspectos e tendências. Revista Brasileira de Estudos de População, v.3, n. 2, p. 87-129, 1986. 


\section{Sobre os autores}

Carlos Lobo é doutor em Geografia pela Universidade Federal de Minas Gerais. Bolsista do Programa Pesquisador Mineiro da Fapemig. Professor adjunto e chefe do Departamento de Geografia da Universidade Federal de Minas Gerais (UFMG). Credenciado nos Programas de Pós-Graduação em Análise e Modelagem de Sistemas Ambientais e em Geografia, do Instituto de Geociências da UFMG. Editor-chefe da revista Geografias, uma publicação do Programa de Pós-Graduação e do Departamento de Geografia da UFMG.

Leandro Cardoso é doutor e mestre em Geografia pela Universidade Federal de Minas Gerais (UFMG). Professor adjunto IV do Departamento de Engenharia de Transportes e Geotecnia da UFMG e coordenador pro tempore do Programa de Pós-Graduação em Geotecnia e Transportes da UFMG.

Ivânia Linhares de Almeida é engenheira e mestre em Geotecnia e Transportes pela Escola de Engenharia da Universidade Federal de Minas Gerais (UFMG). Técnica do Conselho Regional de Engenharia, Arquitetura e Agronomia de Minas Gerais.

Ricardo Alexandrino Garcia é doutor em Demografia pela Universidade Federal de Minas Gerais (UFMG). Professor do Departamento de Geografia do Instituto de Geociências (IGC) da UFMG e coordenador do Laboratório de Estudos Territoriais (Leste/IGC/UFMG). Coordenador do Programa de Pós-graduação em Geografia da UFMG. Editor-chefe do Cadernos do Leste e editor da revista Geografias.

\section{Endereço para correspondência}

\section{Carlos Lobo}

Departamento de Geografia, sala 333 - Instituto de Geociências, Universidade Federal de Minas Gerais

Av. Antônio Carlos, 6627, Pampulha

31270-901 - Belo Horizonte-MG, Brasil

\section{Leandro Cardoso}

Escola de Engenharia, bloco 1, sala 3603 - Universidade Federal de Minas Gerais

Av. Antônio Carlos, 6627, Pampulha

31270-901 - Belo Horizonte-MG, Brasil

Ivânia Linhares de Almeida

Escola de Engenharia, bloco 1, sala 3603 - Universidade Federal de Minas Gerais

Av. Antônio Carlos, 6627, Pampulha

31270-901 - Belo Horizonte-MG, Brasil

\section{Ricardo Alexandrino Garcia}

Departamento de Geografia, sala 333 - Instituto de Geociências, Universidade Federal de Minas Gerais

Av. Antônio Carlos, 6627, Pampulha

31270-901 - Belo Horizonte-MG, Brasil

\section{Abstract}

Commuting and metropolitan interaction/cohesion: a methodological proposal for the municipalities of the Metropolitan Region of Belo Horizonte/MG - 2010

It is not unusual for specific academic literature to contain controversies regarding the limits and actual territorial configuration of the metropolitan regions in Brazil. Aside from the lack 
of consensus and well defined criteria to establish these regional and political-administrative outlines, it seems relevant to consider the meaning of commuting as an indicator of the metropolitan social and economic dynamic. The objective of this article is to investigate the level of integration of the municipalities that make up the Metropolitan Region of Belo Horizonte $(\mathrm{MRBH})$, based on the flows of pendular displacement. With information extracted from the sample micro-data of the Demographic Census 2010, the methodology presented made it possible to propose an Index of Metropolitan Integration for each municipality in the region, derived from the Internal Pendularity Ratio, Pendular Connectivity and Nuclear Pendularity In general, the results highlighted some very low levels of integration, especially in the cases of the municipalities of Itatiaiuçu, Itaguara and Baldim.

Keywords: Integration metropolitan. Commuting. Metropolitan Region of Belo Horizonte.

\section{Resumen}

Movilidad pendular e interacción/coesión metropolitana: uma proposta metodológica para os municipios de la Región Metropolitana de Belo Horizonte/MG - 2010

No é raro na literatura acadêmica, existe controvérsias sobre los límites y la configuración territorial de las regiones metropolitanas no Brasil. Afora a falta de consenso y objetivos bien definidos para los sistemas de recortes regionales y políticos-administrativos, parece importante considerar el significado de la movilidad pendular como indicador de la dinámica económica y social metropolitana. El objetivo de este artículo es investigar el nivel de integración de los municípios que integran una RMBH, teniendo como base los flujos de desplazamientos pendulares. Comisiones extraídas de los Censo Demográficos 2010, una metodología permitida una propuesta para cada municipio de la región de un Índice de Integración Metropolitana, derivada de la Pendularidad Interna, de la Conectividad Pendular y de la Pendularidad Nuclear. En general, los resultados indicaron sensibles diferencias regionales en el espacio metropolitano, lo que permitió destacar algunos niveles muy bajos de integración, especialmente para los casos de los municipios de Itatiáñez, Itaguara y Baldim.

Palabras clave: Integración metropolitana. Movilidad pendular. Región Metropolitana de Belo Horizonte. 
